\title{
Level 1: Was bedeutet eigentlich Gaming?
}

Beginnen möchte ich mit einer kleinen Einführung in das Thema Gaming. Dieses Thema hat in den letzten zehn Jahren massiv an Bedeutung gewonnen. Basierend auf der Erkenntnis, dass Gaming zu unserer kulturellen und gesellschaftlichen Lebensrealität gehört, und dass es zudem neue Formen des Lernens und Arbeitens erlaubt, wird es auch und vor allem für Bibliotheken immer interessanter.

Was ist mit Gaming gemeint? Gaming ist nicht automatisch gleichzusetzen mit Spielen, auch wenn es hier eine sehr große Schnittmenge gibt. Manche Autoren gehen davon aus, dass Gaming quasi eine Untergruppe des Spielens sei. Ich bin nicht dieser Meinung, denn Gaming meint mehr als das Spielen von Computergames. Es geht dabei auch um die entstandene Gaming-Kultur, die Aktivitäten der Spieler außerhalb des eigentlichen Games und die daraus resultierenden neuen Denk- und Arbeitsweisen, inkl. Themen wie Informations- und Wissensmanagement, Arbeitsorganisation etc. Es geht aber auch um Lesen und Schreiben und um Ästhetik und neue Kultur- und Lernorte. Alle diese Elemente sind Teil des Themas Gaming. Aus diesem Grund bin ich der Meinung, dass man von einer großen Schnittmenge zwischen beiden Themen - Gaming und Spielen - ausgehen sollte.

Die Beschäftigung mit dem Thema Spielen bzw. Spiel an sich ist nicht neu. Schon seit mehr als 150 Jahren wird in verschiedenen Studien und Publikationen über die Verbindung von Spielen und unserer kulturellen Lebensrealität nachgedacht. Ein besonders empfehlenswertes Buch zu diesem Thema ist das von Johan Huizinga verfasste Werk „Homo Ludens - Vom Ursprung der Kultur im Spiel“. In diesem Buch verweist er auf elementare Kriterien:

1. Freiwilligkeit

2. Grenzen und Regeln

3. Ein oder mehrere klar definierte Ziele

4. Abgrenzung zum ,realen“ Leben

5. Spannung und Freude

Die US-amerikanische Autorin und Games-Entwicklerin Jane McGonigal beschreibt in ihrem Buch „Reality is broken“ Gaming mittels vier relevanter Kriterien:

1. Ein klares Ziel

2. Ein Regelwerk, welches individuelle Freiräume bietet und Kreativität zulässt

3. Ein starkes Feedback

4. Freiwillige Teilnahme und Akzeptanz der Ziele, Regeln und Feedbacks

Ralph Koster wiederum fokussiert sich in seinem Buch „A Theory of Fun for Game Design“ auf den Faktor „Fun“ bzw. „Spaß“.

Ist damit das Thema Gaming abschließend definiert? Ich denke nicht. Die hier genannten Definitionen beschreiben sicherlich die Struktur von Games, aber es gibt noch mehr relevante Punkte zu beachten. Gerade das Verhältnis zwischen Gaming und Spielen ist interessant, weshalb ich im Folgenden kurz darauf eingehen möchte.

Wie bereits erwähnt, ist Gaming eng verwandt mit Spielen. Man könnte auch sagen, es ist eine Form des Spielens. Und Spielen ist wiederum ein elementarer Bestandteil unserer Kultur, ja sogar unserer Spezies. Einen Großteil der Dinge, die wir in unserem Leben lernen - vor allem in den ersten Lebensjahren - erschließen wir uns durch Spielen. Und selbst im Erwachsenenalter beschäftigen wir uns mit unterschiedlichen Facetten des Spiels. Nehmen wir beispielsweise den Sport. Mannschaftssport- 
arten wie z. B. Fussball oder Handball sind - bei aller Professionalität - Spiele. Und auch das Entwickeln bzw. das Leben in virtuellen Welten hat gerade in Deutschland Tradition. Der Karneval, jene virtuelle Welt, die jedes Jahr auf's Neue das Leben von Millionen von Menschen - nicht nur im Rheinland - verändert und sogar das Leben ganzer Regionen auf den Kopf stellt, ist nichts anderes als ein Spiel. Schaut man genau hin, stellt man fest, dass die eben beschriebenen Definitionen auch hier passen.

Interessant ist in diesem Zusammenhang die Tatsache, dass der Mensch die einzige Spezie ist, die einen Unterschied zwischen Lernen und Spielen macht. Bei allen anderen Kreaturen auf diesem Planeten bedeutet Spielen gleichzeitig auch Lernen und umgekehrt.

Wir wissen also, dass Spielen etwas völlig Normales ist und dass letztlich jeder Mensch auf die eine oder andere Art und Weise spielt. Was aber bedeutet dann in diesem Zusammenhang Gaming?

Eli Neiburger, Assistant Director der District Library Ann Arbor, hat auf seiner Vortragsreise durch Deutschland im Frühjahr 2013 auf ein paar wesentliche Punkte hingewiesen: Für ihn liegt ein wesentlicher Unterschied in den Maschinen, die wir für Gaming benutzen. Sie ermöglichen zweierlei: Zum Einen können Maschinen virtuelle und komplexe Welten erschaffen, die über die Möglichkeiten unserer Gehirne hinausgehen. Mit den Maschinen sind unserer Phantasie nun wirklich keine Grenzen mehr gesetzt. Zum Anderen können wir durch diese Technologien Tausende von Menschen im Spiel miteinander vernetzen. Die Technologie erweitert also die Möglichkeiten bzw. das Spektrum des Spiels. Ich stimme Eli Neiburger zu und möchte zudem noch auf zwei weitere Punkte hinweisen: Die Technologien, die wir nutzen um Computergames zu spielen, sind immer auch Teil der aktuellen Medien- und Kommunikationswelt. In vielen Fällen stellen Games den ersten Kontakt mit einem Computer, einem Tablet-PC oder einem Smartphone dar. Meines Erachtens hätten sich viele unserer aktuellen Kommunikations- und Medientechnologien nicht durchsetzen können, wenn man mit ihnen nicht auch spielen könnte. Gaming bzw. Elemente davon sind zudem eine spezielle Herangehensweise beim Erlernen der Nutzungsfunktionen moderner Technologien. Try and Fail, Vernetzen, Feedback etc. ersetzen in zunehmenden Maße klassische Gebrauchsanweisungen. Gaming wirkt also bei sehr vielen Menschen als Motivationsquelle für das Erlernen bzw. Nutzen moderner Kommunikations- und Medientechnologien.

Auch wenn diese Definitionen abstrakt erscheinen mögen, ist es wichtig, dass Sie sich damit beschäftigen. Sie werden merken, dass nur wenige Menschen verstehen, was Gaming bedeutet. Die von mir angesprochenen Merkmale sollten Sie am besten mit Beispielen beschreiben. Gerade wenn Sie gegenüber Ihrem Träger oder kritischen Eltern erklären müssen, warum Sie sich als Bibliothek intensiv mit Gaming beschäftigen möchten, sollten Sie in der Lage sein, die wesentlichen Punkte zu erläutern. Besonders hilfreich ist immer die Verbindung zu anderen spiel-ähnlichen Aktivitäten in unserer Gesellschaft, wie z.B. dem Karneval. Diese alte Tradition ist durchaus vergleichbar mit der Mechanik von Computerspielen bzw. virtuellen Welten. Damit nehmen Sie dem Thema seine Brisanz.

\section{Gaming - eine kulturelle Erfolgsgeschichte}

Bevor wir auf die Geschichte der Computergames eingehen, möchte ich etwas Wichtiges anmerken. Die Geschichte der Computergames ist sehr komplex und zudem sehr vielschichtig. Es gibt zur Geschichte der Computergames eine Vielzahl an spannenden Büchern, und es ist unmöglich und zugleich wenig zielführend, im Rahmen eines

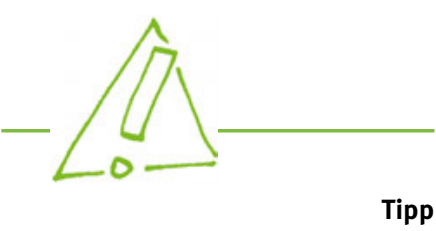

Auch wenn diese Definitionen helfen mögen, ist es wichtig, dass Sie selber zum Gamer werden und einzelne Spiele zumindest ausprobieren. 
Buches zum Thema „Gaming in Bibliotheken“ die gesamte Geschichte der Computergames in ihrer Breite darzustellen. Aus diesem Grund gebe ich Ihnen hier nur einen kleinen Einblick in die Historie. Der Fokus liegt dabei auf einem grundsätzlichen Verständnis der Geschichte der Computergames und nicht auf einer umfassenden Abhandlung des Themas. Denjenigen von Ihnen, die mehr über die historischen Hintergründe des Themas erfahren möchten, empfehle ich zwei Quellen:

1. Das Buch „The Ultimate History of Video Games: from Pong to Pokemon and beyond ... the story behind the craze that touched our lives and changed the world" von Steven Kent

2. Die „Timeline“ des Computerspielemuseums in Berlin. Dabei handelt es sich um eine animierte Onlineplattform, welche bedeutende historische Fakten zum Thema Gaming zur Verfügung stellt.

\section{Eine kleine Gaming-Geschichte}

Der Siegeszug der Computergames begann mit einer sehr einfachen Maschine: der „Odysee“ des US-amerikanischen Unternehmens „Magnavox“. Diese 1972 auf dem Markt erschienene und von Ralph Baer entwickelte Konsole erweiterte die Nutzungsmöglichkeiten des sich gerade als Massenmedium durchsetzenden Fernsehens. Die Spiele waren sehr einfach und die Maschine konnte damals noch keine komplexen Welten darstellen. Alles, was die Spieler bekamen, war ein Controller und zwei Balken auf dem Fernseher zwischen denen ein Punkt hin- und herbewegt werden sollte. Es war also eine einfache Art „Tennis“, die jedoch die Welt nachhaltig verändern sollte. Man spielte diese Spiele übrigens nicht alleine sondern immer zu zweit bzw. in Gruppen. Gaming war also schon immer ein soziales Kulturphänomen bzw. eine soziale Aktivität.

Es dauerte nicht lange, und es wurden weitere Konsolen entwickelt und veröffentlicht. Mit Pong (Atari 1975), für welches Atari lange Zeit Lizenzgebühren an Magnavox zahlen musste, da es sich letztlich um eine Adaption des Spiels der Odysee handelte, wurde Gaming in den Wohnzimmern zu einem Massenphänomen. Nur wenig später kamen auch die ersten mobilen Gaming-Konsolen auf den Markt. Das USamerikanische Unternehmen Mattel veröffentlichte 1976 mit „Football“ die erste mobile Gaming-Konsole. Mit dem Aufkommen der ersten Heimcomputer, wurden auch Spiele für diese Systeme entwickelt. Nach dem TV wurde also nun auch der Computer mit Gaming erweitert. Zu Anfang waren es vor allem Computer der Firma Apple, auf denen man spielte. Später sollten Computer mit dem Betriebssystem Windows von Microsoft zur wichtigsten und erfolgreichsten Plattform für PC-Games werden.

Mit der Weiterentwicklung der Konsolen war es möglich, das Spiel von der Konsole zu trennen. Am Anfang waren Spiel und Konsole ein System. Später jedoch war es möglich, die Spiele auszutauschen. Sie wurden auf Modulen geliefert, die man in die Konsolen steckte. Dadurch war es möglich geworden, dass Unternehmen Spiele für Konsolen entwickeln konnten, ohne selber für die Hardware verantwortlich zu sein.

Ein weiteres Erfolgskriterium waren die überall entstehenden Spielhallen. In ihnen konnte eine Vielzahl an Videogames gespielt werden. Viele Klassiker wie z.B. Pac Man, Donkey Kong oder Asteroids waren ursprünglich „Automatenspiele“. Da es aber möglich geworden war, dass man die Spiele der Konsolen austauschen konnte, wurden nicht nur neue Spiele entwickelt, sondern auch die Spielhallen-Klassiker für die Konsole adaptiert. Die Kultur der Spielhallen ging in Deutschland leider wieder zugrunde. In manchen asiatischen Ländern und mit etwas Glück auch in den USA kann man solche einzigartigen Räume aber noch finden. 


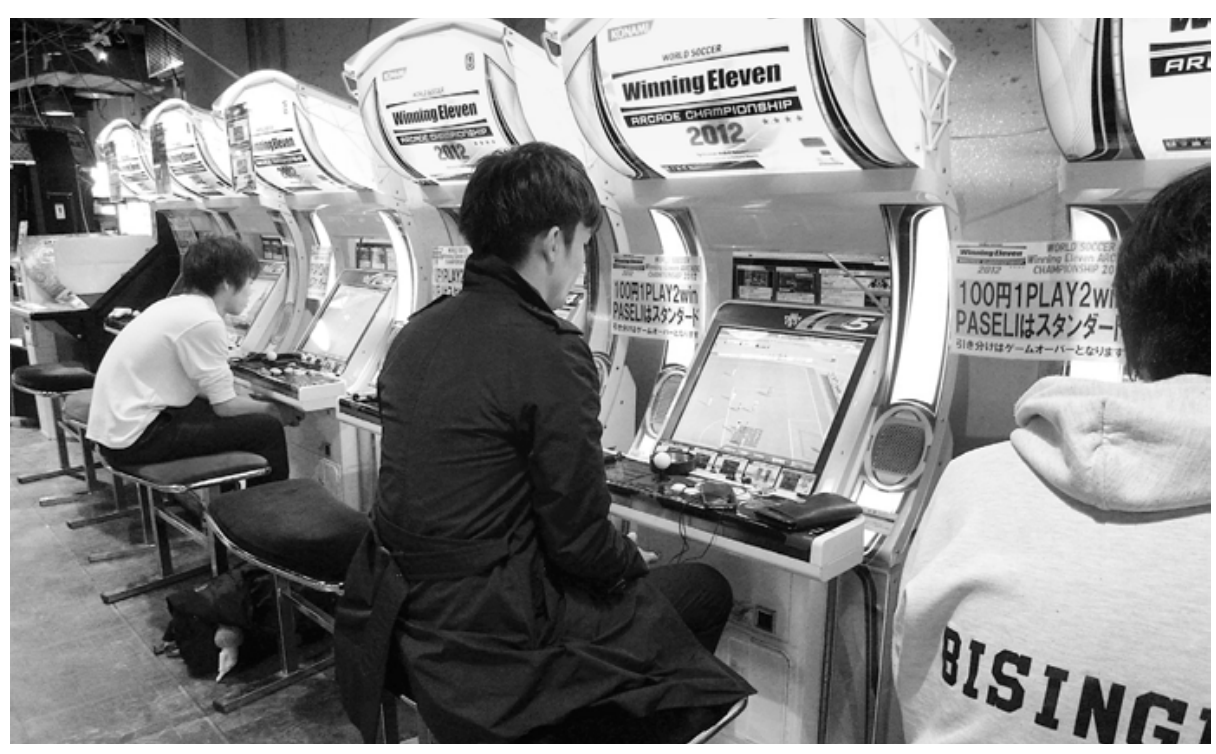

Abb. 2 Arcade in Tokio/Japan.

In den folgenden Jahren wurden immer neue Konsolen und Computer und damit verbunden immer neue Spiele veröffentlicht. Mit zunehmender Rechenleistung konnten die Spiele immer komplexer und graphisch anspruchsvoller werden. Viele von Ihnen werden sich mit Sicherheit noch an den Commodore C64 oder aber die Konsole Nintendo NES erinnern. Beide wurden ein großer Erfolg. Die Nintendo NES kam 1985 auf den Markt und war bereits 1990 in jedem dritten US-Haushalt zu finden. Mit dem „Mega Drive“ von Sega und dem Nintendo Super NES war der nächste Sprung zu 16Bit-Konsolen vollzogen worden.

Der Markt änderte sich rasant. Viele Unternehmen verschwanden und andere kamen neu hinzu. Mit der Vorstellung des Game Boy 1989 läutete Nintendo ein neues Zeitalter ein. Mit diesem Gerät konnte sich Nintendo für sehr lange Zeit eine Vormachtstellung sichern. 1994 kam dann die Playstation von Sony auf den Markt. Sie revolutionierte die Welt der Konsolen u. a. mit der Tatsache, dass es hierfür keine Module sondern CD-ROMs gab, auf denen die Spiele gespeichert wurden. Besonders interessant waren auch die verschiedenen Zusatzgeräte wie z.B. der DualShock-Controller, der vibrieren konnte und damit ein noch intensiveres Spielgefühl ermöglichte. In zunehmendem Maße wurde nun damit begonnen, nicht nur Bild und Ton sondern weitere Sinne anzusprechen bzw. in das Spielerlebnis zu integrieren.

\section{Gaming heute}

Im Laufe der letzten 20 Jahre hat sich die Gaming-Welt kontinuierlich weiterentwickelt. Im Bereich der Konsolen gibt es heute drei relevante Anbieter: Sony (Playstation 1-4, Playstation Portable, Playstation Vita), Microsoft (XBOX, ХВOX360, XBOX One) und Nintendo (Wii, Wii U, Nintendo DS, Nintendo 3DS) ${ }^{1,2}$ Im Bereich der Heimcom-

1 Die Playstation4 und die XBOX One sind zum Zeitpunkt der Erstellung dieses Buches noch nicht auf dem Markt. Ich habe sie aber auf der Gamescom 2013 testen können - großartig!

2 In einigen Fällen gibt es noch weitere Varianten der genannten Konsolen. Ich habe hier nur die wesentlichen benannt. 
puter ist Windows nach wie vor das am meisten benutzte und zudem auch am besten funktionierende Betriebssystem. Allerdings gibt es zunehmend auch Angebote für die Betriebssysteme OS und Linux. Die Firma Valve möchte ein eigenes Betriebssystem speziell für Gaming entwickeln. Mit dem Aufkommen der Smartphones und TabletPCs, wie z.B. dem iPad, sind neue und sehr erfolgreiche Gaming-Plattformen auf den Markt gekommen. Dies merken vor allem die Anbieter von mobilen Konsolen, wie dem Nintendo DS und der Playstation Vita. Auch die Peripherie hat sich weiterentwickelt. Neue Arten von Controllern bis hin zu der Tatsache, dass der Körper des Spielers zum Controller wird, sind ebenso auf dem Vormarsch wie neue Visualisierungstechnologien. Mit Themen wie Gamification, Augmented Reality Games, Alternate Reality Games etc. wird wiederum die Mechanik von Computergames in andere Zusammenhänge z. B. für Game-Based-Learning übernommen. Darauf komme ich später zurück.

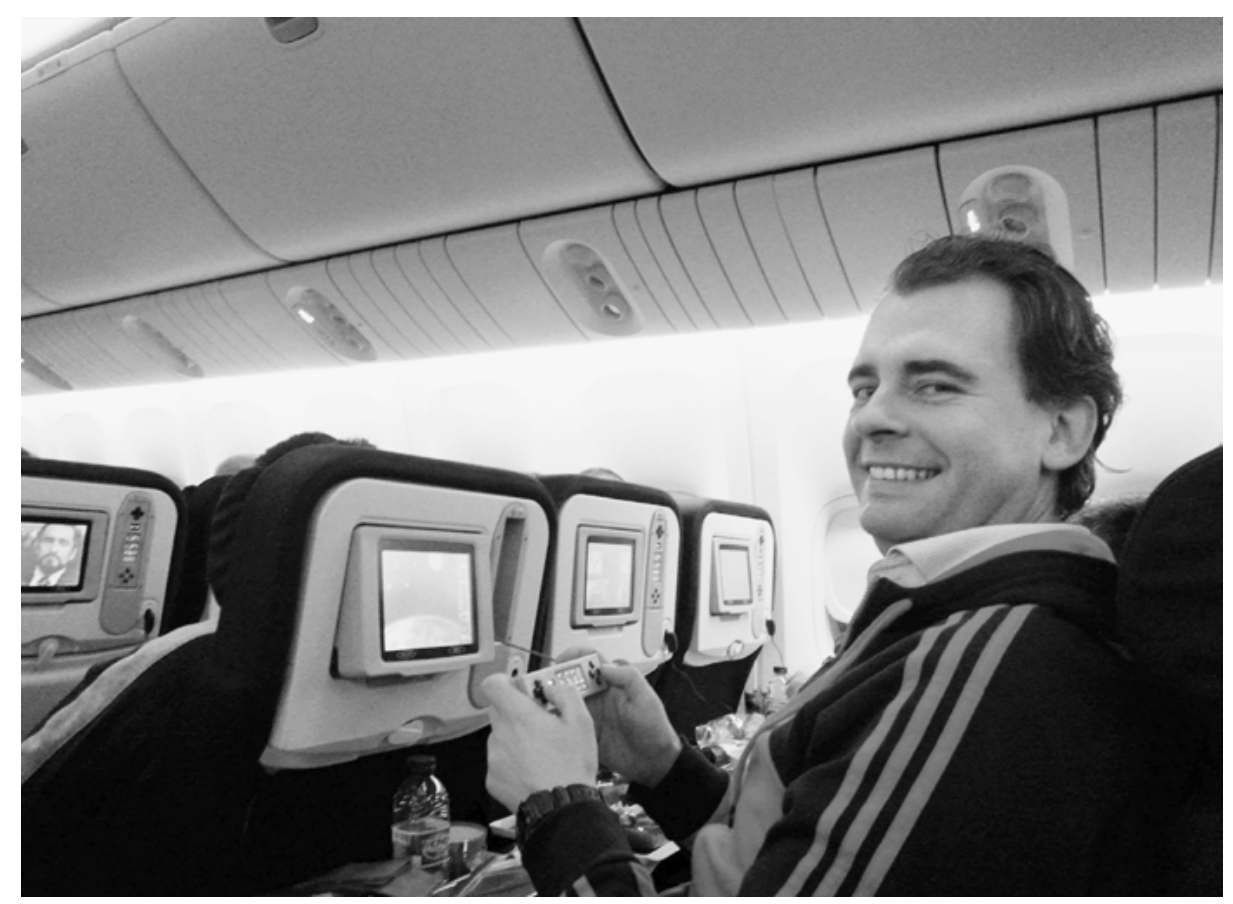

Abb. 3 Gaming im Flugzeug - auf dem Weg nach Hongkong.

Gaming ist und bleibt eine Erfolgsgeschichte. Es ist bis heute keine Verlangsamung der Entwicklung zu erkennen. Gewiss, es gab immer wieder Krisen und Rückschläge. Diese hatten aber nur einen Einfluss auf Unternehmen aber nicht auf das Interesse der Gamer.

Aktuell gibt es sicherlich kaum eine Aktivität, die rund um den Globus so viele Menschen begeistert und vernetzt wie das Spielen unterschiedlicher Computerspiele. Ein paar Beispiele gefällig? Sehr gerne:

1. Würde man alle gespielten Stunden von World of Warcraft zusammenzählen und in Jahre ausdrücken, so würde man auf die unglaubliche Zahl von 5.930.000 Jahre kommen $^{3}$ - und World of Warcraft ist ein vergleichsweise kleines Spiel. Der Wiki zum Spiel hat übrigens über 200.000 Seiten.

3 Quelle: „Reality is broken“ von Jane McGonigal 
2. Spieler, genauer gesagt Kinder, die Pokemon spielen, lernen durch ein einziges Spiel mehr neue Worte kennen als in den ersten beiden Schuljahren.

3. Gaming bzw. eSports haben einen wesentlichen Anteil daran, dass Südkorea zum größten Kultur-Exporteur der Welt aufgestiegen ist.

4. Auf der alljährlich stattfindenden Gaming-Messe „Gamescom“ in Köln kommen hundertausende Besucher zusammen, um „ihre“ Spiele zu feiern.

5. In Deutschland spielen mehr als $25 \%$ aller Einwohner Computerspiele - Tendenz steigend. Das durchschnittliche Alter eines Gamers bzw. einer Gamerin in Deutschland ist 32. Der Frauenanteil beträgt 50\%.

Besuchen Sie unbedingt die Gamescom. Dies ist kein Scherz. Wenn Sie die Gamescom besuchen, können Sie auf einfache Art und Weise einen tiefen Einblick in die aktuelle technische und inhaltliche Situation der Gaming-Welt bekommen. Zudem erleben Sie „live“, was Gaming bedeuten kann.

Fragen Sie erwachsene Bibliotheksnutzer, ob sie sich noch an ihre Zeit als Gamer erinnern können und welche Hard- und Software benutzt wurde. 